\title{
Cellular Level Imaging of the Retina Using Optical Coherence Tomography
}

\author{
Cherry Greiner and Stacey S. Choi \\ New England College of Optometry \\ USA
}

\section{Introduction}

Low-coherence interferometry was first demonstrated, in the early 1990s, in imaging the eye due its tissue transparency and minimal attenuation of the input light. For this reason, ophthalmic imaging is the most successful application of optical coherence tomography (OCT). Since its first demonstration, advancements in technology and OCT designs have resulted in higher resolution images of the retinal layers including retinal photoreceptors, retinal pigment epithelium (RPE), and retinal nerve fiber layer (RNFL) thus improving the potential for early diagnosis, the understanding of disease pathology, as well as the assessment of therapeutic response. This chapter will mainly discuss improvements in OCT systems over time, particularly developments towards cellular level imaging and their clinical applications in ophthalmology in the present and in the future.

The success of OCT is in part due to its ability to obtain high structural information at reasonable depths, bridging the capabilities of confocal and ultrasound imaging. OCT is analogous to ultrasound pulse echo imaging, using light instead of acoustic waves. Since the signal from OCT is traveling considerably fast, i.e. at the speed of light, OCT detects optical echoes from tissue surfaces using low coherence interferometry technique. As a result, higher resolution cross-sectional images of tissue structures are possible compared to ultrasound imaging (Fercher et al., 1988; Fujimoto et al., 1986). Early OCT images were based on time domain approaches with axial and lateral resolutions of less than $20 \mu \mathrm{m}$ (Huang et al., 1991; Swanson et al., 1993). Interests in OCT increased resulting in the use preliminary in clinical studies and eventually to the development of commercial OCT system for ophthalmic imaging in the mid-1990s. The development of high speed scanners increased imaging speed capabilities of time domain OCT (TD-OCT). Nonetheless, dramatic improvements in scan speed was not attained until the development of Fourier domain detection, i.e. spectral domain (SD-OCT) or swept source OCT. By removing the need to mechanically scan the reference mirror increased scan speeds by an order of magnitude from a few thousand to ten to hundred thousand axial scans per second while also improving sensitivity (de Boer et al., 2003; Lietgeb et al., 2003, Potsaid et al., 2008). In addition to speed and sensitivity, other important parameters that should be considered for cellular level imaging are high axial and lateral resolutions.

Developments of wider band light sources $\geq 80 \mathrm{~nm}$, which we referred to as "ultrawideband" in this chapter, have dramatically improved the axial resolution to $\leq 5 \mu \mathrm{m}$ 
at $800 \mathrm{~nm}$. In addition, ultrawideband light source at other wavelengths, such as those over $1000 \mathrm{~nm}$, provide structural imaging beyond the retinal layers (Povazay et al., 2003; Puvanathasan et al., 2008). The lower scattering at longer wavelengths is an important design consideration since it can extend the applicability of OCT technique to certain patient populations with pre-existing afflictions (Povazay et al., 2003). In parallel to light source developments to improve axial resolutions, researchers continue to improve lateral resolutions in OCT ophthalmic imaging. A vital technological advancement in improving lateral resolution in OCT is the use of adaptive optics (AO) to correct for ocular aberrations. Multiple variations of AO-OCT currently exist; as AO-OCT systems continue to develop, they offer great clinical potential for improved clinical assessment of retinal pathologies great clinical potential for improved clinical assessment of retinal pathologies.

OCT systems have proven its effectiveness in detecting small retinal changes in patients with various retinal diseases from age-related macular degeneration (AMD) to retinal detachment. Hence it has become an integral part of diagnostic paradigm in clinical practice in ophthalmology. By incorporating ultrawideband source and AO in OCT systems, it is now possible to resolve individual photoreceptors as well as nerve fiber bundle. The power of cellular level in-vivo imaging is that it allows clinicians to follow retinal and optic nerve changes over time in patients, which ultimately results in earlier detection and intervention. Current applications of both OCT and AO-OCT will be reviewed and future direction will be discussed.

\section{Ultrahigh resolution OCT designs}

\subsection{Ultrawideband light sources}

For ophthalmic imaging, high axial resolution in OCT images is particularly important due to the stratified organization of the retina. In OCT, the interference signal detected is the field autocorrelation of the light source. Since the envelope of the autocorrelation is the Fourier transform of the source power spectrum, the axial resolution is determined by coherence length, $1_{c}$, of the light source:

$$
\Delta z=l_{c}=\frac{2 \ln 2 \lambda_{c}^{2}}{\pi \Delta \lambda}
$$

where $\lambda_{c}$ is the center wavelength and $\Delta \lambda$ is the spectral bandwidth. Axial resolutions can therefore be improved by using lower wavelengths and/or increasing the spectral bandwidth. In selecting the choice of wavelength, it is also important to consider tissue absorption and scattering that influence OCT imaging contrast and penetration depth. Hemoglobin and melanin are main tissue absorbers that limit penetration depth of wavelengths in the visible to near infra-red regime. At longer wavelengths but less than $1800 \mathrm{~nm}$, tissue scattering dominates, which decreases with increasing wavelength. At wavelength greater than $1800 \mathrm{~nm}$ and around $1400 \mathrm{~nm}$, imaging is limited by the absorption of water. For these reasons and available laser at certain wavelengths, OCT typically uses 800 and $1300 \mathrm{~nm}$ center wavelengths. Ultrawideband sources such as superluminscent diodes (SLD), femtosecond Titanium:Sapphire (Ti:Sapph) lasers and photonic crystal fiber (PCF) based sources, are typically designed in either wavelength regime. Another important consideration is the attainable resolution for a given spectral bandwidth at a given center 
wavelength. Fig. 1 shows the required optical bandwidths to achieve axial resolutions at 800 and $1300 \mathrm{~nm}$. Assuming similar spectral shape, the axial resolution is better with $800 \mathrm{~nm}$ light source for the same spectral bandwidth. In addition, the shallower slope for $1300 \mathrm{~nm}$ compared to $800 \mathrm{~nm}$, indicates that the use of longer wavelength will require a larger increase in bandwidth in order to reach the same axial resolution as with shorter wavelengths.

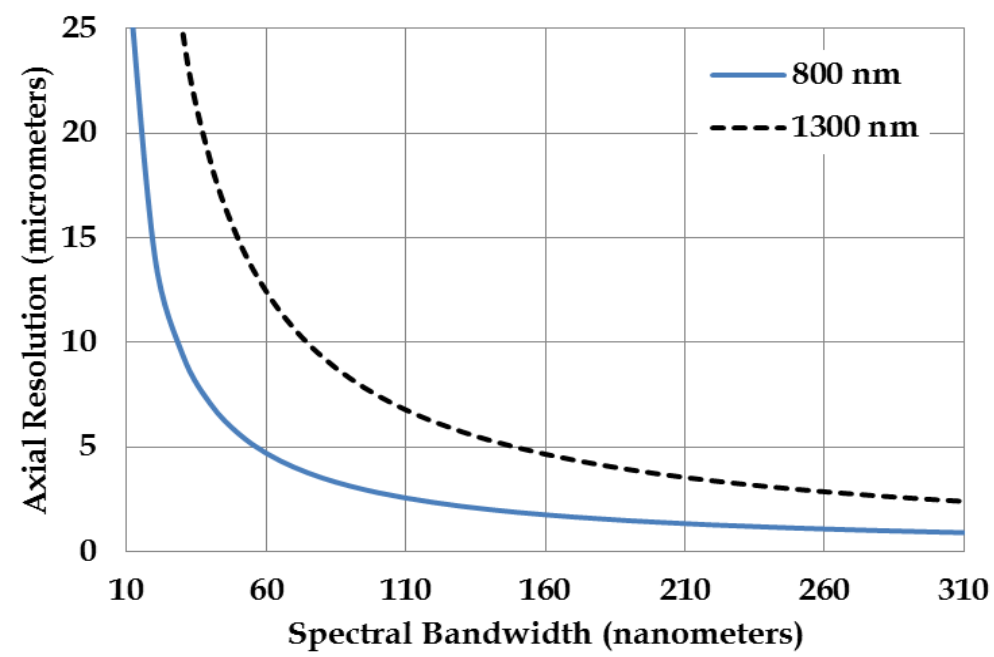

Fig. 1. Comparison of optical bandwidths and axial resolutions at two center wavelengths, 800 and $1300 \mathrm{~nm}$.

\subsubsection{SLD sources}

Light source for conventional OCT imaging is typically SLDs with spectral bandwidths of 20-30 nm resulting in OCT axial resolutions of 10-15 $\mu \mathrm{m}$. Though this resolution is sufficient to image retinal layers, identification of individual cells or sub-cellular structures is not possible. Pushing towards cellular imaging, advanced SLDs have been developed with wide spectral bandwidths $\geq 80 \mathrm{~nm}$ by multiplexing 2 or more SLDs, providing OCT axial resolutions of $<10 \mu \mathrm{m}$ (Adler et al., 2004; Cense et al., 2009a; Hong et al., 2007; Ko et al., 2004; Potsaid et al., 2008; Zawadzki et al., 2005). A prototype of this ultrawideband SLD for OCT imaging was first demonstrated by Ko et al. in 2004. Two independent SLD sources at center wavelengths 840 and $920 \mathrm{~nm}$ were combined with a custom designed fiber coupler based on fiber GRIN microlenses and miniature dielectric coated mirrors (Adler et al., 2004; Ko et al., 2004). OCT imaging in the retina with this SLD, which had a $155 \mathrm{~nm}$ bandwidth, resulted in axial resolutions of $\sim 3.2 \mu \mathrm{m}$. Fig. 2 shows a comparison between images obtained with an ultrawideband SLD OCT system and a conventional commercial system from the same subject. As shown, multiple retinal layers were better defined with the ultrawideband SLD. Moreover, retinal features such as photoreceptor segments, external limiting membrane and ganglion cell layers were better visualized (Ko et al., 2004). With a $170 \mathrm{~nm}$ SLD source, more choroidal vessels were visualized, allowing one to find connecting vessels between adjacent imaging areas (Hong et al., 2007). 

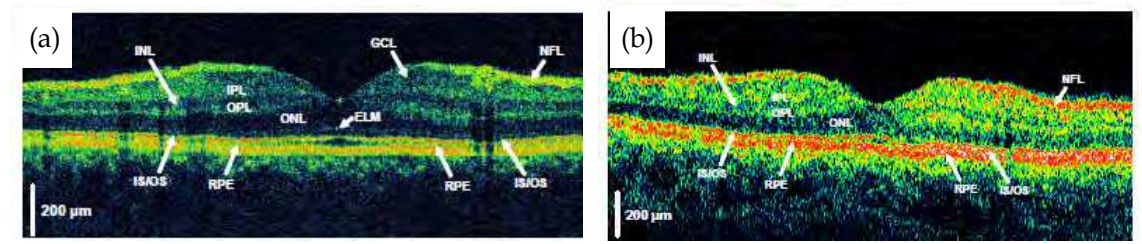

Fig. 2. Comparison of in-vivo images acquired with (a) an OCT sytem with an ultrawideband SLD source with an axial resolution of $3.2 \mu \mathrm{m}$ and(b) a standard resolution OCT with an axial resolution of $10 \mu \mathrm{m}$. Images from Ko et al., 2004.

One of the drawbacks of SLD is that its spectral shape can be less Gaussian-like and more spectrally modulated. As a result, the axial point spread function (PSF) has sidelobes that can result in image artifacts. Despite this limitation, spectral shaping may be applied digitally to approximate a Gaussian envelope and reduce the sidelobes (Potsaid et al., 2008; Tripathi et al., 2002). SLDs, however, offer advantages over other sources, which can be key in transitioning high resolution OCT systems from the laboratory and into the clinic. In particular, they are highly efficient, reliable and portable. Moreover, SLDs are lower in cost, as much as an order of magnitude in comparison to femtosecond lasers.

In assessing the imaging quality between SLDs and femtosecond sources, a comparative study has recently been performed by Cense et al. (2009). They used a Femtolasers Integral Ti:Sapph laser with a spectral bandwidth, center wavelength and power of $135 \mathrm{~nm}, 800 \mathrm{~nm}$, and $60 \mathrm{~mW}$, respectively (Cense et al., 2009a). In comparison, the BroadLighter, a multiplexed SLD, has $110 \mathrm{~nm}$ spectral bandwidth, $840 \mathrm{~nm}$ center wavelength and $12 \mathrm{~mW}$ of input power (Cense et al., 2009a). The output spectrums from each source are shown in Fig. $3 a$. Both sources were used on the same OCT system and imaged the same patient on different days. In-vivo measurements showed similar axial resolutions in tissue specifically, $3.2 \mu \mathrm{m}$ with the Ti:Sapph laser and $3.3 \mu \mathrm{m}$ with the SLD (Fig. 3b) (Cense et al., 2009). Despite more pronounced sidelobes in the axial PSF with the SLD compared to that with the Ti:Sapph laser (Fig. 3b), the difference in the dynamic range was $\leq 5 \mathrm{~dB}$, which the group expected to be within the range of variability between individual measurement sessions (Cense et al., 2009). Finally, a comparison of acquired volumetric images showed that the wideband SLD can image individual cone photoreceptors, retinal capillaries and nerve fiber bundles as well as the Ti:Sapph laser source (Fig. 3c-d) (Cense et al., 2009).

(a)

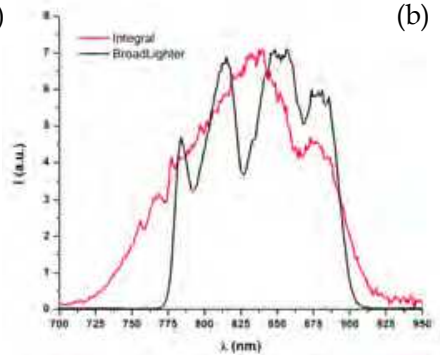

(b)

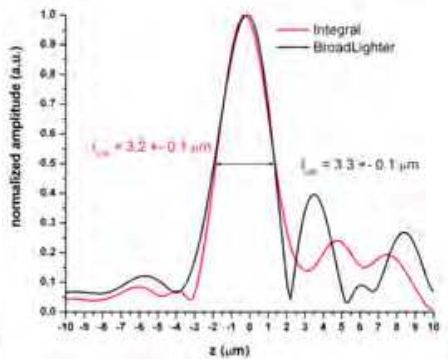


(c)

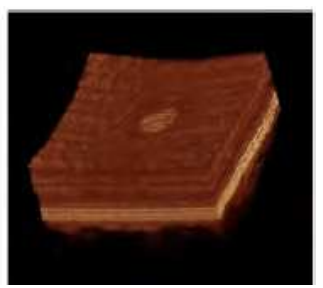

(d)

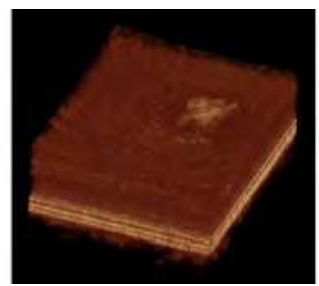

Fig. 3. (a) Comparison of spectra from ultrawideband Ti:Sapph (Integral) and multiplexed SLD (BroadLighter). (b) Normalized coherence function from both sources. (c,d) Comparison of high resolution imaging with the ultrawideband SLD (c) and Ti:Sapph (d) light sources. Images from Cense, et al., 2009.

\subsubsection{Titanium:Sapphire laser sources}

Since OCT was developed in research laboratories, it is not surprising that the first sub-10 $\mu \mathrm{m}$ axial resolution OCT imaging was demonstrated using a laboratory source, femtosecond Ti:Sapph laser, as opposed to a commercial SLD source (Clivaz et al., 1992). Femtosecond mode-locked lasers can generate not only wide spectral bandwidth, but also provide sufficient power for high speed imaging. A novel design is a high power Kerr-lens modelocked Ti:Sapph laser with a spectral bandwidth of up to $350 \mathrm{~nm}$ at $800 \mathrm{~nm}$ center wavelength developed by Drexler et al. (1999). High performance was obtained with custom designed chirped mirrors with high reflective bandwidth and intracavity dispersion compensating prims (Drexler et al., 1999). In-vivo ophthalmic imaging of a normal subject showed axial resolutions of $\sim 2-3 \mu \mathrm{m}$ (Drexler et al., 2001; Wojtkowski et al., 2004). Later, improvements resulted in less complex, more compact and lower cost Ti:Sapph laser with 135-176 nm spectral bandwidth (Unterhuber et al., 2003). Spectral broadening was accomplished by specially designed low dispersive mirrors and the footprint was reduced by asymmetric folding of the resonator and some double pass configurations (Unterhuber et al., 2003). In-vivo imaging of patients with macular pathologies showed images with $\sim 3 \mu \mathrm{m}$ axial resolutions (Unterhuber et al., 2003).

\subsubsection{PCF based sources}

Another class of wideband light sources for high resolution OCT imaging is based on nonlinear propagation of pulsed laser into PCF. PCFs are optical fibers made with pure silica core surrounded by air holes throughout the length of the fiber. The large difference in refractive index between the air and the silica leads to nonlinear effects resulting in supercontinuum generation, i.e. extreme broadening of narrow band incident pulse into a spectrally broadband continuous output. Just a few pulses with nJ of energy are sufficient to generate supercontinuum (Humbert et al., 2006). Moreover, fiber dispersion can be engineered to control temporal broadening. Hartl et al. (2001) reported the first supercontinuum based OCT system. A femtosecond Ti:Sapph laser was launched into a PCF resulting in a spectral bandwidth of $450 \mathrm{~nm}$ in the $1300 \mathrm{~nm}$ wavelength region resulting in an axial resolution of 2 $\mu \mathrm{m}$ in tissue (Hartl et al., 2001). Even higher resolutions have been demonstrated. Modifications including fiber lengths, core sizes and polarization states can be used to optimize the performance of PCF based sources (Apolonski et al., 2002). With some optimization, a PCF based source was developed with a spectral bandwidth $\sim 400 \mathrm{~nm}$ centered 
at $750 \mathrm{~nm}$ resulting in an axial resolutions of $\sim 0.5 \mu \mathrm{m}$ (Povazay et al., 2002). OCT crosssectional imaging was demonstrated using monolayers of human colorectal adenocarcinomas on coverslips with OCT images showing subcellular structures, such as the nucleoli (Povazay et al., 2002). Humbert et al. (2006) later demonstrated that supercontinuum can also be generated by launching Ti:Sapph laser into tapered PCF. Controlled tapering involved the heating and stretching of a section of the fiber forming a narrow waist (Birks et al., 2000). The decrease in the fiber pitch or distance between the air holes from the tapering process increases the non-linear process (Humbert et al., 2006). Using tapered PCF, a maximum spectral bandwidth of $194 \mathrm{~nm}$ centered at $809 \mathrm{~nm}$ was developed, resulting in OCT images with an axial resolution of $1.5 \mu \mathrm{m}$ in air (Humbert et al., 2006).

OCT imaging with PCF based light source centered at $1100 \mathrm{~nm}$ has also been demonstrated. This wavelength regime is of interest since the absorption of melanin in RPE decreases with wavelength, therefore imaging with wavelengths greater than $800 \mathrm{~nm}$ should allow for better imaging of the choroid below the RPE layer. In a study by Povazay et al., (2003) a Ti:Sapph laser was launched into a PCF generating a supercontinuum source from 400 to $1200 \mathrm{~nm}$ which was then passed through long pass filter, resulting in a spectrum with a $165 \mathrm{~nm}$ bandwidth centered around $1050 \mathrm{~nm}$ (Povazay et al., 2003). OCT images of ex-vivo pig retina were acquired and also compared with OCT images using a $165 \mathrm{~nm}$ bandwidth Ti:Sapph laser at $800 \mathrm{~nm}$ (Povazay et al., 2003). As expected, better visualization of intraretinal layer was noted with the lower center wavelength source, i.e. Ti:Sapph laser (Fig. 4a) (Povazay et al., 2003). However, the PCF based light source showed improved penetration in the choroid, as evident by more visible choroidal blood vessels as shown in Fig. 4 b (Povazay et al., 2003).

(a)

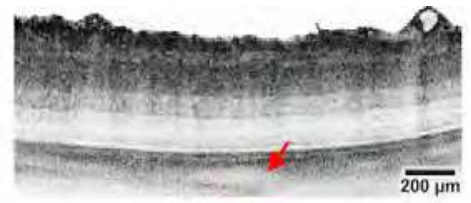

(b)

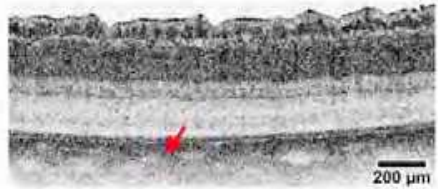

Fig. 4. $(a, b)$ Comparison of OCT images acquired with (a) a Ti:Sapph laser and (b) a PCF fiber source. Red arrows indicates blood vessels in the choroid. Images from Povazay, et al., 2003.

More recently, $1064 \mathrm{~nm}$ was pumped into PCF generating both 800 and $1300 \mathrm{~nm}$ wavelengths simultaneously. Near complete depletion of the pump wavelength created a double peak spectrum with bandwidth and average power of $116 \mathrm{~nm}$ and $\sim 30 \mathrm{~mW}$, respectively at $800 \mathrm{~nm}$ center wavelength and $156 \mathrm{~nm}$ and $\sim 48 \mathrm{~mW}$ at $1300 \mathrm{~nm}$ center wavelength (Aguirre et al., 2006). However, coupling loss reduced the power by nearly $50 \%$ (Aguirre et al., 2006). Additionally, power stability was found to be sensitive to environmental condition (Aguirre et al., 2006). Fiber end facet protection was recommended to improve long term power stability (Aguirre et al., 2006). The supercontinuum source was integrated into a TD-OCT system with different couplers, sample arm optics and photodiode detector for each center wavelength but with a common galvometric scanner and scanning stage (Aguirre et al., 2006). OCT imaging in hamster cheek pouch showed axial resolutions of $2.2 \mu \mathrm{m}$ at $800 \mathrm{~nm}$ and $4.7 \mu \mathrm{m}$ at $1300 \mathrm{~nm}$. Some blurring of the image was observed at both wavelengths due to side lobe coherence artifacts on the PSF as a result of spectral modulation at 800 and $1300 \mathrm{~nm}$ (Aguirre et al., 2006). To address this issue, further work will investigate spectral smoothing (Aguirre et al., 2006). 
Another dual band OCT imaging was demonstrated with 840 and $1230 \mathrm{~nm}$ center wavelength with $1060 \mathrm{~nm}$ pump wavelength (Spoler et al., 2007). The spectral shape of the supercontinuum source was tailored with a double pass prism, razor blades and an end mirror (Spoler et al., 2007). Resulting bandwidths were $>200 \mathrm{~nm}$ after spectral filtering with $>100 \mathrm{~mW}$ average power for each wavelength (Spoler et al., 2007). The source was incorporated in a free space OCT system to launch both wavelengths simultaneously (Spoler et al., 2007). Dichroic filters separated the two wavelength channels prior to detection (Spoler et al., 2007). In-vitro OCT imaging of a rabbit eye was performed and showed axial resolutions <2 and < $4 \mathrm{um}$ at 840 and $1230 \mathrm{~nm}$ in tissue, respectively (Spoler et al., 2007). As expected, $840 \mathrm{~nm}$ imaging yielded higher resolution while higher penetration was achieved with the longer $1230 \mathrm{~nm}$ wavelength (Fig. 5a \& 5b) (Spoler et al., 2007). Simultaneous measurements allowed the feasibility to extract spectroscopic information, since differences in image contrast are influenced by wavelength dependent scattering and absorption of different types of tissue (Spoler et al., 2007). Differences in intensities are used to construct spectroscopy images (Fig. 5c) that provided tissue differentiation, such as the conjunctiva and sclera (Spoler et al., 2007).
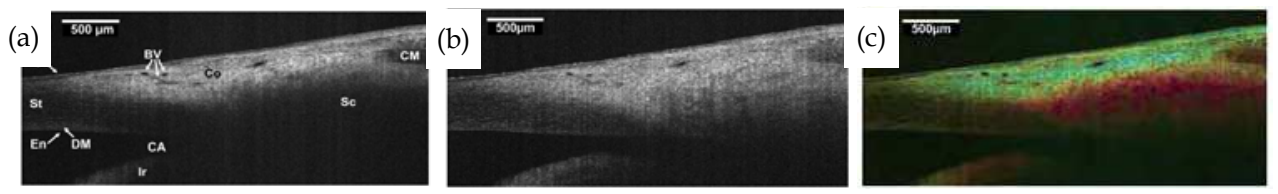

Fig. 5.(a-c) OCT imaging at (a) $840 \mathrm{~nm}$ and (b)1230 nm (c) Differential color image based on scattering intensity from each wavelength range. Higher scattering in $840 \mathrm{~nm}$ is shown in shades of blue while those $1230 \mathrm{~nm}$ is in shades of red. Ep: Epithelium; BV: Blood vessel; CM: Ciliary muscle; St: Stroma; Sc: Sclera; En: Endothelium; DM: Descement's membrane; CA: Chamber angle; Co: Conjunctiva. Images from Spoler et al., 2007.

\subsection{Adaptive Optics (AO)}

In OCT, axial and lateral resolutions are decoupled. The lateral resolution in OCT is similar to that in standard microcopy:

$$
\Delta x=\frac{4 \lambda f}{\pi D}
$$

where $\mathrm{f}$ is the focal length of the focusing lens and the $\mathrm{D}$ is the beam diameter on the lens. Therefore, lateral resolution can be improved by increasing the numerical aperture of the beam delivery optics by decreasing the focal length and/or increasing the beam diameter. In ophthalmic imaging, the focusing optics is limited by the eye itself. A $3 \mathrm{~mm}$ pupil diameter will yield a lateral resolution of $10-15 \mu \mathrm{m}$ diffraction limited spot at $800 \mathrm{~nm}$ center wavelength (Drexler, 2004). Increasing the pupil diameter by $1 \mathrm{~mm}$ will reduce the focused beam spot diameter to $4.3 \mu \mathrm{m}$, in theory. However with larger pupil size, the ocular aberrations will limit the achievable size of the focus beam. Additionally, the use of broadband light sources to improve axial resolution will further contribute to chromatic aberrations. 
Adaptive optics (AO) in OCT has been shown to be a successful approach in correcting ocular and system's aberrations to improve lateral resolutions. The two essential components of an $\mathrm{AO}$ system for retinal imaging are a wavefront sensor and a corrector placed conjugate to the pupil plane of the eye as shown in Fig. 6a. To measure ocular aberrations, a Shack- Hartmann wavefront sensor containing a 2D array of lenslets, each of which with the same diameter and focal length is used. Light reflected from the retina are distorted by aberrations of the eye particularly from corneal surfaces and crystalline lens (Yoon et al., 2006). This reflected light is spatially sampled by the 2D lenslet array on the wavefront sensor, forming multiple beams focused at the focal plane of the lenses and imaged onto a camera. Light reflected from a perfect eye, i.e., free of aberrations, emerges as a collimated beam. Therefore, Shack-Hartmann spots will be imaged at the focal point of each lenslet, forming a regularly spaced grid (Fig. 6b, left). In comparison, wavefront error from a real eye, i.e., with aberrations such as defocus and astigmatism, will displace the Shack-Hartmann spots from the optical axis of each lenslet, resulting in an image of an irregular grid (Fig. 6b, right). Since the displacement is proportional to the wavefront slope sampled by the lenslet at a particular location, the aberrations from the eye can be determined. The aberration information is then used to alter the phase profile of the incident wavefront by changing the physical length that the light propagates to correct the wavefront error. Different types of wavefront correctors or deformable mirrors (DMs) have been used including those based on bimorph, piezoelectric and micro-electro-mechanical systems (MEMS) technologies (Doble \& Miller, 2006). An example of a MEMs based mirror with piston/tip/tilt capability per mirror segment is shown in Fig. 6c.
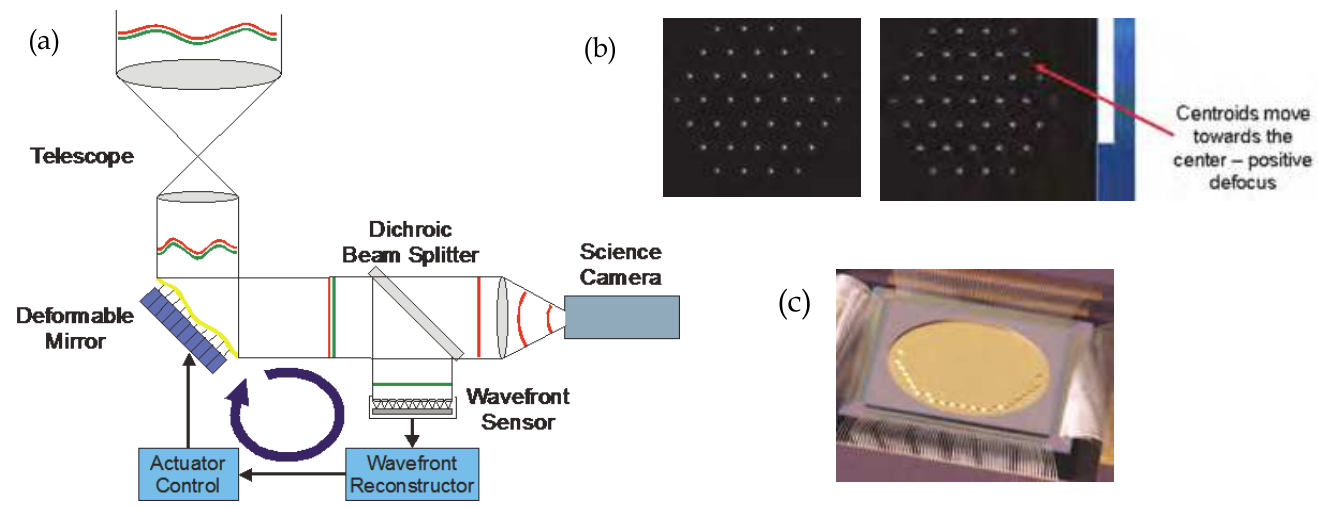

Fig. 6.(a)A schematic of an AO system for retinal imaging. (b) Example of a ShackHartmann wavefront sensor images from a perfect eye (left) and from a real eye (right).

(c) Scanning electron microscope of a 163 segment MEMs based mirror. Images courtesy of Iris $\mathrm{AO}$, Inc.

The first attempt to improve retinal images from scanning laser ophthalmoscope with AO was shown by Dreher et al. (1989). Using a DM, the group corrected the astigmatism in one eye based on the patient's spectacle prescription (Dreher et al., 1989). Several years later, the Hartmann-Shack wavefront sensor was implemented to measure the wavefront error of the eye (Liang et al., 1997). With the DM, aberrations from the system and the eye were also corrected (Liang, 1997). Initially, wavefront compensation was achieved with a closed loop 
feedback system. At each loop, images were acquired and the aberrations of the system and eye were calculated. The DM partially, $\sim 10 \%$, corrected the wavefront error and loop iterations were performed until a minimum wavefront error was reached. Later, closed loop $\mathrm{AO}$ systems were developed with faster and automated wavefront sensing and dynamic correction of ocular aberrations (Hofer, 2001; Fernandez 2001). To date, AO has been successfully combined with ophthalmic imaging systems such as flood illuminated fundus camera, scanning laser ophthalmoscope (SLO) and OCT systems for improved lateral resolution and image contrast (Choi et al., 2005; Choi et al., 2006; Jonnal et al., 2007; Roorda et al., 2002a; Roorda et al., 2002b; Thorn et al., 2003; Wolfing et al.,2006; Xue et al.,2007).

\subsubsection{AO with ultrawideband source OCT}

Cellular level OCT imaging can be realized by combining AO with ultrawideband sources to achieve high lateral and axial resolutions. Hermann et al. (2004) first applied AO with a $130 \mathrm{~nm}$ bandwidth Ti:Sapph laser in a TD-OCT system. With AO, the Strehl ratio was improved by at least a factor of 10 (Hermann et al., 2004). The axial resolution with this system was $3 \mu \mathrm{m}$ while the lateral resolution was $\sim 5-10 \mu \mathrm{m}, 2-3$ times better than similar systems without AO (Hermann et al., 2004). In later developments, AO was combined with SD-OCT systems, taking advantage of the higher sensitivity, enabling faster data acquisition. Fig. 7 shows an example design of a SD-OCT system with an AO sub-system.

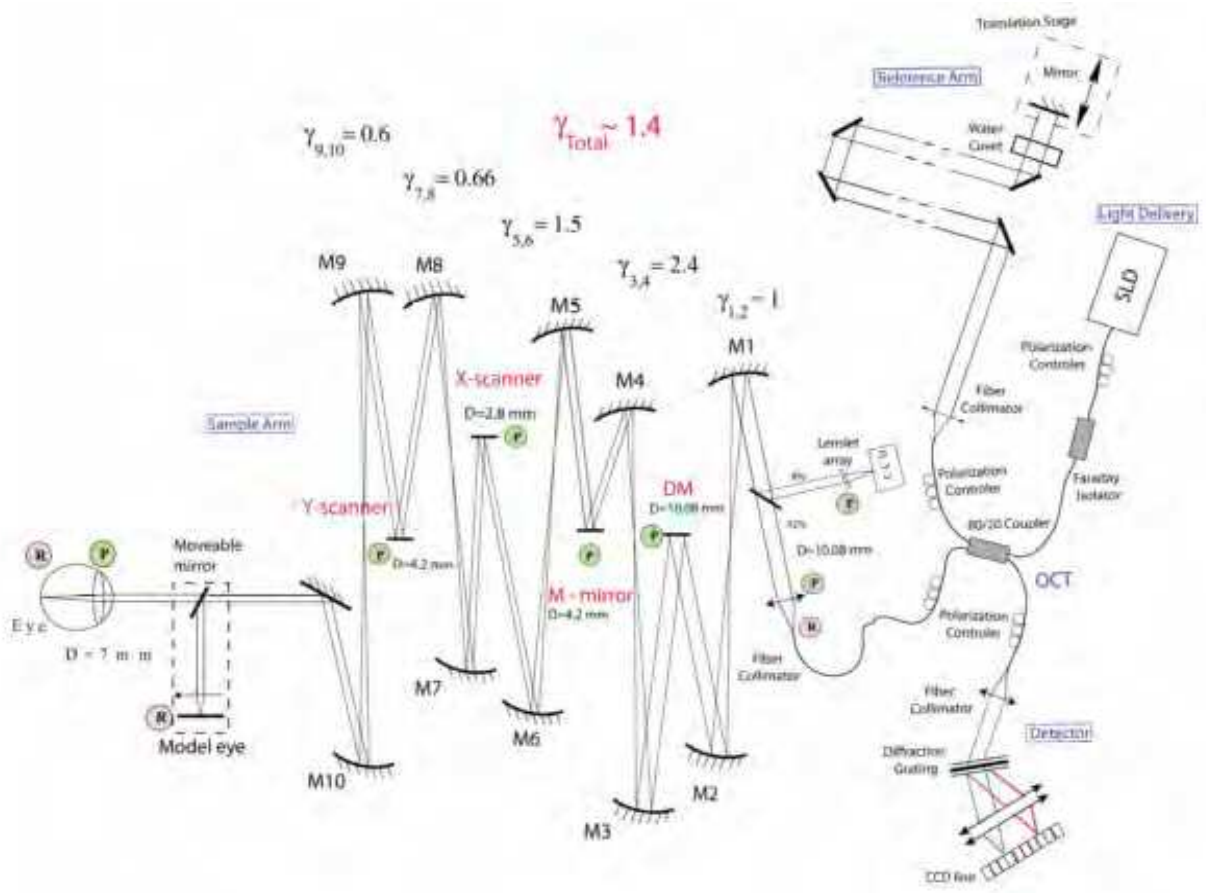

Fig. 7. A schematic of an AO-OCT system. P: Pupil plane; R: Retinal plane; M: Spherical mirror; $\gamma$ : Telescope magnification. Image from Zawadzki et al., 2005. 
The system was designed with a series of telescopes that relayed the pupil conjugate plane to the Shack Hartmann, bimorph DM and two scanning mirrors, while the retinal conjugate plane is relayed to the OCT detector. The SLD source used had a spectral bandwidth of 140 $\mathrm{nm}$ centered at $890 \mathrm{~nm}$. Axial and lateral resolutions were $6 \mu \mathrm{m}$ and $4 \mu \mathrm{m}$, respectively. With $\mathrm{AO}$, it was found that image brightness was improved which the group attributed to be due to astigmatism and defocus correction. Overall improvements allowed for better visualization of retinal structures and cone photoreceptors (Zawadzki et al., 2005).

Although AO-OCT systems are mostly used to image the photoreceptor layer, it can also be used to image the RNFL, which is important in the clinical management of glaucoma. With an AO-OCT system, Kocaoglu et al. (2011) were able to easily visualize individual axonal bundles in both enface and cross-sectional views due to improved image contrast, as a result of wavefront error correction. For the first time, the researchers were able to quantify individual nerve fiber bundles, i.e. width and thickness, from acquired OCT images (Kocaoglu et al., 2011).

\subsubsection{Dual-DM AO-OCT systems}

Since wavefront correctors have a limited dynamic range, the use of trial lenses may be necessary particularly in correcting subjects with large refractive errors. Others, however, have developed alternative designs to increase the range of wavefront correction of AOOCT systems. One example is the addition of a Badal to coarsely correct for large defocus (Chen et al., 2008). Another design alternative is to incorporate two wavefront correctors with the first DM (DM1) mainly used to correct for large stroke, low order aberrations while the second DM (DM2) used to correct for low stroke, high order aberrations (Cense et al., 2009; Mujat et al., 2010; Zawadzki et al., 2007,2008). DM1 is first used to correct for aberrations, as calculated by the wavefront sensor, then held static after reaching minimum wavefront error. The remaining aberrations are again determined by the wavefront sensor, and then corrected by DM2. Fig. 8 shows the time course of the dual DM AO implementation. Though DM1 corrected most of the aberrations, these were further reduced after both DMs were implemented (Zawadzki et al., 2007).
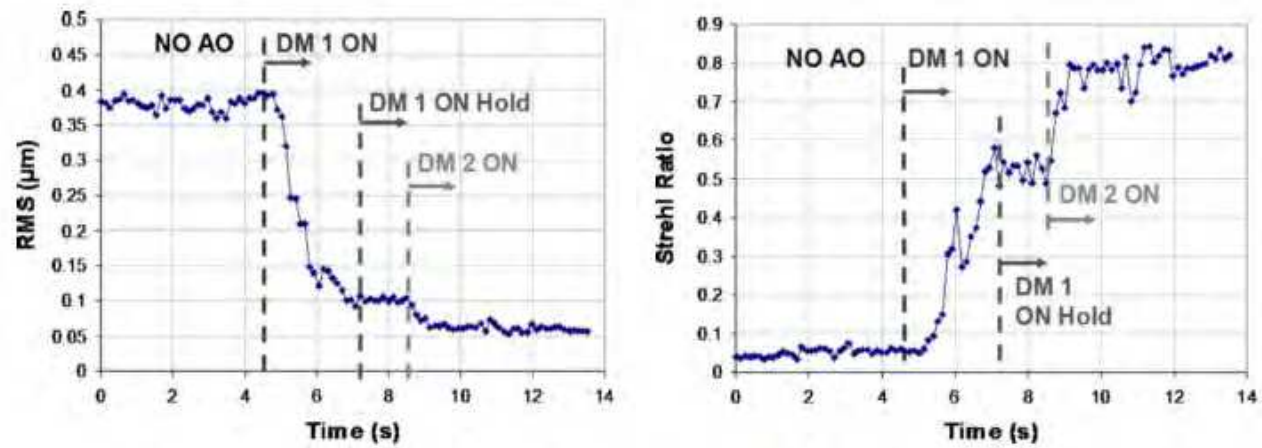

Fig. 8. Time course comparing RMS (left) and Strehl ratio (right) during dual-DM AO implentation. Images from Zawadzki et al., 2007. 


\subsubsection{Correcting motion artefacts in AO-OCT}

Despite faster image acquisition, involuntary head and eye movements still occur, despite the use of bite bars and head restraints, resulting in motion artefacts that distort volumetric OCT images. Though motion artefacts can be corrected via post-processing software, hardware based implementations have also been demonstrated. For example, Fergueson et al. were able to track and correct for retinal motion during or after data acquisition using a low power tracking beam projected onto a retinal feature, such as a blood vessel junction (Ferguson et al., 2004). Real time signal processing is used to determine the position and orientation of the targeted retinal feature to correctly steer the tracking beam (Ferguson et al., 2004). Since the OCT imaging and the tracking beam share the same pair of scanners, the OCT beam similarly follows the retina (Ferguson et al., 2004).

More recently, an alternative system design to correct for motion artifact was demonstrated by Zawadzki et al. by integrating SLO with an AO-OCT. In this combined system, SLO and OCT retinal images were acquired simultaneously, since both modalities share the same AO sub-system and vertical scanner, as shown in the optical schematic diagram, Fig. 9a (Zawadzki et al., 2009). In this system, separate light sources are used for the OCT and SLO optical paths. The SLO imaging beam is combined into and filtered out of the OCT imaging path using dichroic filters. Similarly, a dichroic filter is used to separate SLO and OCT signal in the detection path. To determine motion, the scanning frequencies are such that an SLO frame is acquired for every OCT B-scan, therefore the researchers were able to correlate features in the SLO images with disruption of layers from the B-scan images due to lateral and transverse eye motion (Zawadzki et al., 2009). Fig. 9 b-d show an example of a coregistered AO-OCT B-scan and AO-SLO frame (Zawadzki et al., 2009).

(a)

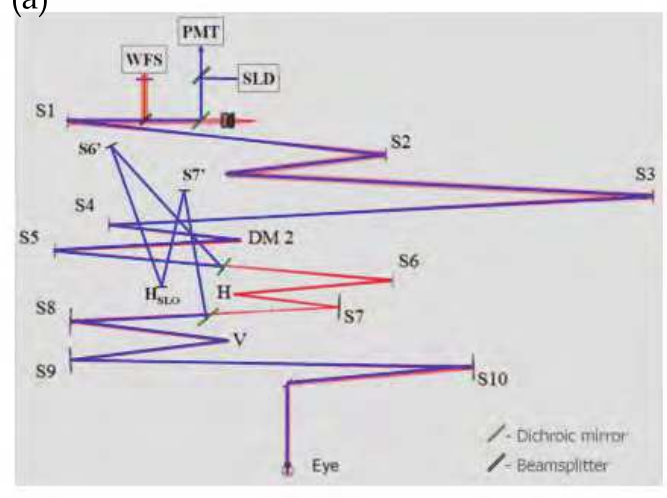

(b)

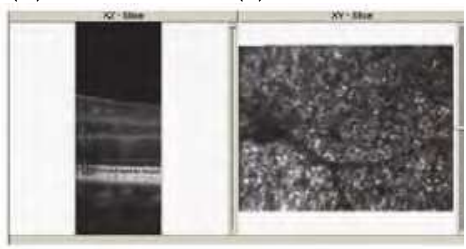

(d)

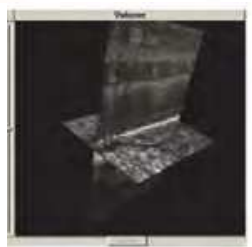

Fig. 9. (a) Optical schematic diagram of combined AO-SLO-OCT system. (b-d) Example of a B-scan (b)acquired at the same time as a SLO frame (c) and resulting co-registered image from both modalities (d). Images from Zawadzki et al., 2011.

Another variation of the combined SLO-OCT system, but without AO, is based on interlacing spectrally encoded SLO frame with OCT B-scans. In spectrally encoded SLO, the illumination beam is dispersed by a diffractive element that spectrally encodes each spatial position (Tao, 2010). The back scattered light is then recombined and directed into a fiber, 
which acts as a confocal detection pinhole (Tao et al., 2010). Spatial information is then decoded by a spectrometer, the same detector used for OCT imaging (Tao et al., 2010). A schematic diagram of the system is shown in Fig. 10. The slow galvanometer of the OCT scan $(\mathrm{Gy}, \mathrm{S})$ acts as a switch between the spectrally encoded SLO (red) and the OCT path (blue), directing the beam towards the combined path (green) (Tao et al., 2010). As shown, the same light source and detector are used by both imaging modalities. During SLO imaging, the galvanometer switch is flipped to its maximum angle, through the grating then along the OCT optical path (Tao et al., 2010). The driving signals for the scanning mirrors are such that a SLO frame is interlaced with an OCT B-scan (Fig. 10 b-c) (Tao et al., 2010). De-interlacing of SLO and OCT frames were performed prior to image registration (Tao et al., 2010). Although this imaging technique has yet to be demonstrated with AO sub-system or the use of ultrawideband light sources, this design has the advantages of potentially allowing for motion tracking while minimizing cost and footprint.
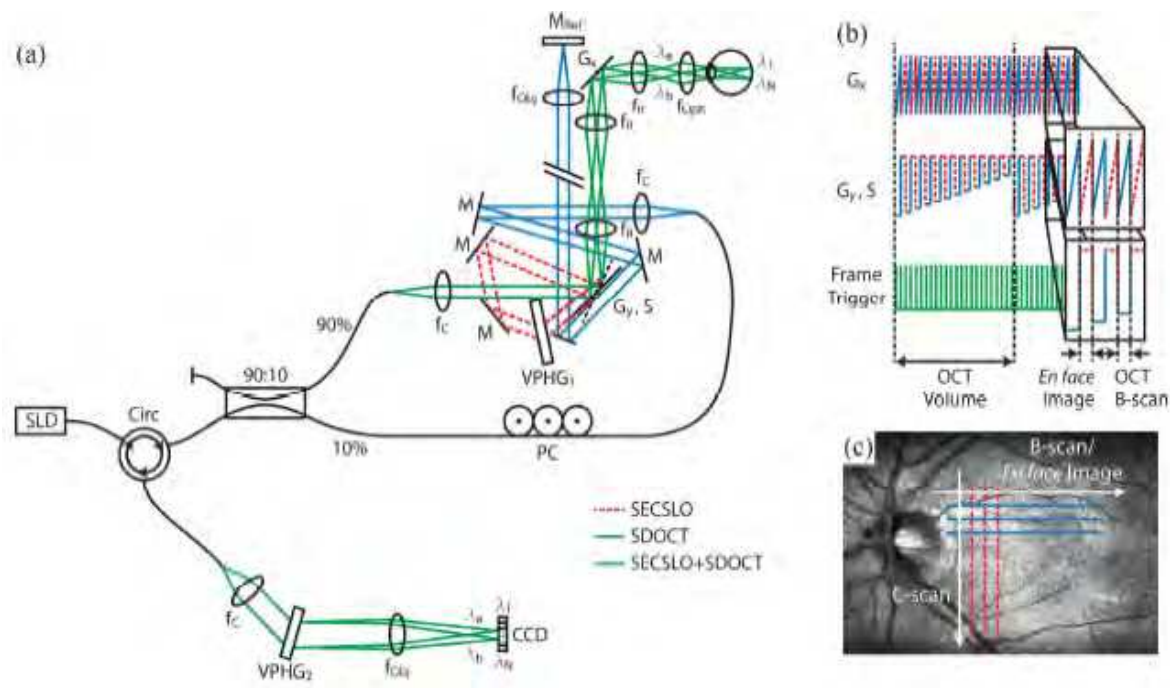

Fig. 10. (a) Optical schematic diagram of combined spectrally encoded SLO and OCT system. (b) Timing diagram showing interlacing of SLO and B-scan images. (c) Illustrated scanning geometry. f: lens, VPHG: Grating, G: Galvanometer, M: Mirror. Images from Tao et al., 2010.

\subsubsection{Correcting chromatic aberrations in AO-OCT}

In spite of the efforts for increasing transverse resolution, AO-OCT systems are still limited in correcting for axial (or longitudinal) and lateral (or transverse) chromatic aberration of the eye, particularly in dilated pupils in combination with the use of broader spectral bandwidth sources. Axial chromatic aberration is manifested as a change in the focus plane with wavelength, while lateral chromatic aberration appears from off-axis illumination laterally shifting the focus with wavelength. The use of an achromatizing lens to correct for axial chromatic aberration was first proposed by Fernandez et al. (2006). This concept was later demonstrated in AO-OCT systems with wide spectral bandwidth sources (Fernandez et al., 2008, Zawadzki et al., 2008). Custom design achromatizing lens was integrated by 
Fernandez et al. in a conjugate pupil plane between the scanning mirrors and the DM, while Zawadzki et al. placed the achromatizing lens immediately after collimating the input laser. Both designs showed improved imaging of the individual photoreceptors with the addition of the achromatizing lens. Fernandez et al. showed that with chromatic aberration correction, photoreceptor structures between the inner and outer segment junctions appear elongated, as opposed to rounded as in standard AO, resembling axial morphology of cones in histology.

Although, an achromatizing lens can correct for axial chromatic aberrations, it does not correct for lateral chromatic aberrations. Zawadzki et al. (2008) theoretically investigated the extent of lateral chromatic aberration and its impact on retinal imaging. The group found that the main contributors to this type of aberration are errors in the lateral positioning of the eye and off-axis imaging (Zawadzki et al., 2008). Proper alignment of the eye relative to the OCT camera optical axis should result in lateral chromatic aberration free imaging (Zawadzki et al., 2008). Based on their system design, the group expects that lateral chromatic aberration is smaller than the lateral resolution that can be achieved by the AO in their system and therefore have little impact on image quality (Zawadzki et al., 2008).

\subsubsection{AO in functional-based OCT systems}

Adaptive optics has also transitioned into functional extensions of OCT, specifically polarization sensitive OCT (PS-OCT). PS-OCT measures the intensity and polarization state in tissue, thus allowing depth resolve imaging of birefringent retinal structures such as the RNFL. The PS-OCT system designed by Cense et al. (2009b) utilized a dual DM AOsubsystem. The group deduced the improvement in signal contrast and resolution with AO allowed for a more sensitive measurement of change in phase retardation and hence birefringence (Cense et al., 2009b). With this system, the group was able to quantify birefringence of thin retinal nerve fiber bundles in the macula lutea, not measurable with standard PS-OCT (Cense et al., 2009b). As AO becomes widely implemented, it is expected that more $\mathrm{AO}$ will be integrated in other forms of OCT.

\section{Clinical applications}

OCT has become an integral part of routine diagnostic paradigm in ophthalmology. Due to its non-invasive nature and the ability to extract quantitative morphological information, it has been extensively used in the diagnosis of retinal and optic nerve diseases as well as in the monitoring of their progression with treatments over time. Here, only some of the selected representative papers on limited topics are briefly reviewed due to space limitation.

\subsection{Ultrahigh resolution OCT}

OCT has been able to reveal various microscopic changes ranging from macular edema to disruptions in photoreceptor layer in patients with various retinal diseases and reduction of RNFL in patients with glaucoma and other types of optic neuropathy.

\subsubsection{Macular edema}

OCT imaging is readily used to diagnose and follow macular edema. It occurs in many disorders including diabetic retinopathy, age-related macular degeneration, retinal vein 
occlusion, uveitis and macular hole. Macular edema is the result of an accumulation of fluid in the retinal layers around the fovea with an increase in retinal thickness, which contributes to vision loss. Fluorescein angiography (FA) has been and still is the critical diagnostic tool for macular edema by identifying the characteristic stellar pattern of cystoid macular edema (CME). Fig. 11 illustrates the appearance of CME through different diagnostic modalities, namely, conventional fundus photography, FA and OCT.
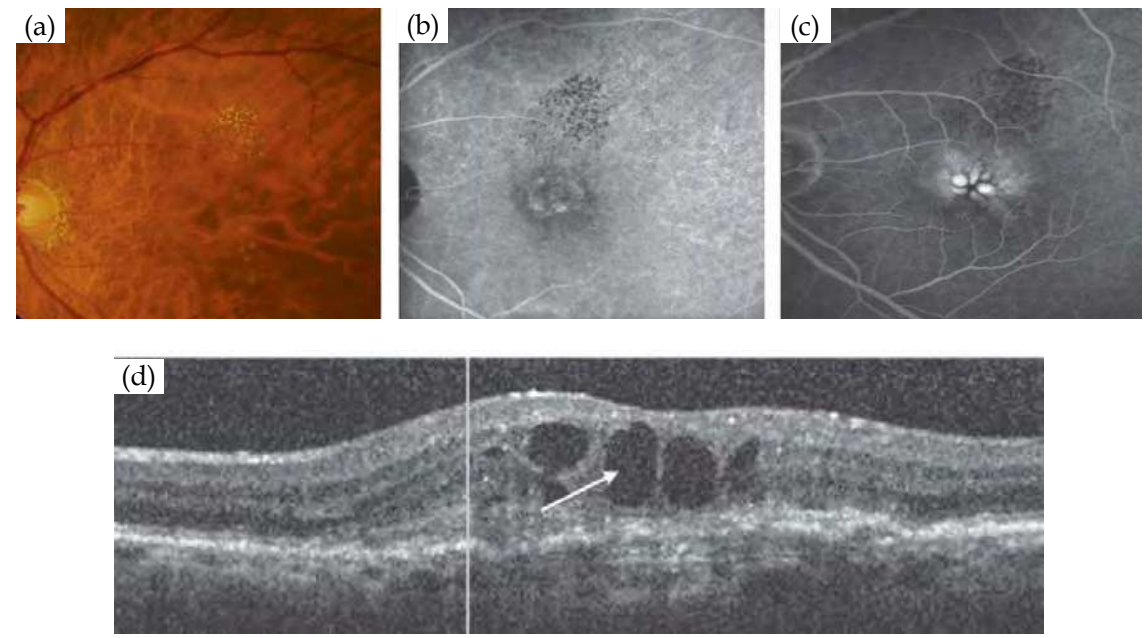

Fig. 11. Cystoid Macular Edema (CME) imaged by 3 different diagnostic modalities. (a) Conventional fundus picture $(b, c)$ Fluorescein angiography (early and late stages respectively) - dilation and leakage of capillary are revealed. Fluorescein dye pools in cystoid spaces and arranged radially from the fovea. (d) OCT image - cystoid spaces are shown. The central macula is indicated with an arrow. Images from Cunha-Vaz, et al., 2010.

In the early stage of macular edema, a diffuse swelling of the outer retinal layer is observed, and then leads to the development of cystoid spaces. In the later stage, the large cystoid spaces can extend from the RPE to the internal limiting membrane, which then eventually rupture causing macular holes. In diabetic patients, macular edema involves three structural changes - most commonly, sponge-like retinal swelling (88\%), then CME (47\%) and then serous retinal detachment (15\%) (Ontani et al., 1999). Fig. 12 shows the fundus pictures and corresponding OCT images of retinal swelling and serous retinal detachment seen in diabetic macular edema.

In sponge-like retinal swelling, the OCT image showed low reflective areas in the outer retinal layers with relatively preserved inner retinal layer with interspersed low reflective areas. The inner retinal layers were displaced anteriorly by the swollen outer retinal layers. In serous retinal detachment, the OCT image clearly showed detached retina as a highly reflective line with the formation of sub-retinal space underneath.

A good correlation has been found between OCT images and visual acuity and FA findings (Hee et al., 1998; Jaffe \& Caprioli, 2004; Ontani et al., 1999). Larger area of increased retinal thickness and the involvement of the macular area correlated with greater loss of vision. Fig. 13 shows the correlation curve between visual acuity (VA) and retinal thickness 

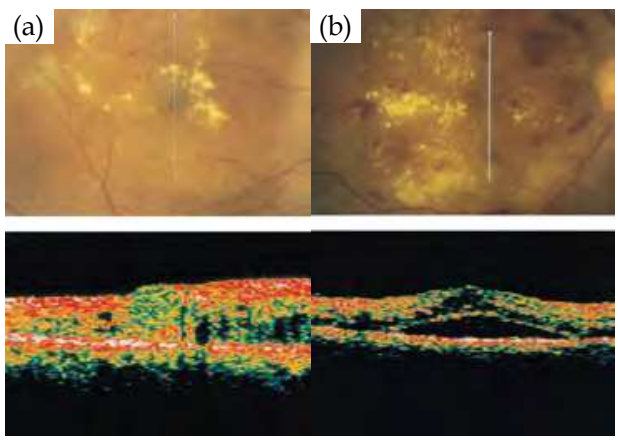

Fig. 12. (a) Retinal swelling. The retinal thickness at the fovea is $560 \mu \mathrm{m}$. (b) Serous retinal detachment with retinal swelling. The thickness of the sub-retinal space is $420 \mu \mathrm{m}$. The vertical arrow on the fundus picture indicates the line and direction of scanning. Scan length $=5 \mathrm{~mm}$. Images from Otani, et al., 1999 .

at the fovea in the eyes with CME (Ontani et al., 1999). Twenty eight of 59 eyes (47\%) developed CME, and the foveal retinal thickness and the VA were found to be negatively correlated with the correlation coefficient of $-0.64, \mathrm{P}<0.01$.

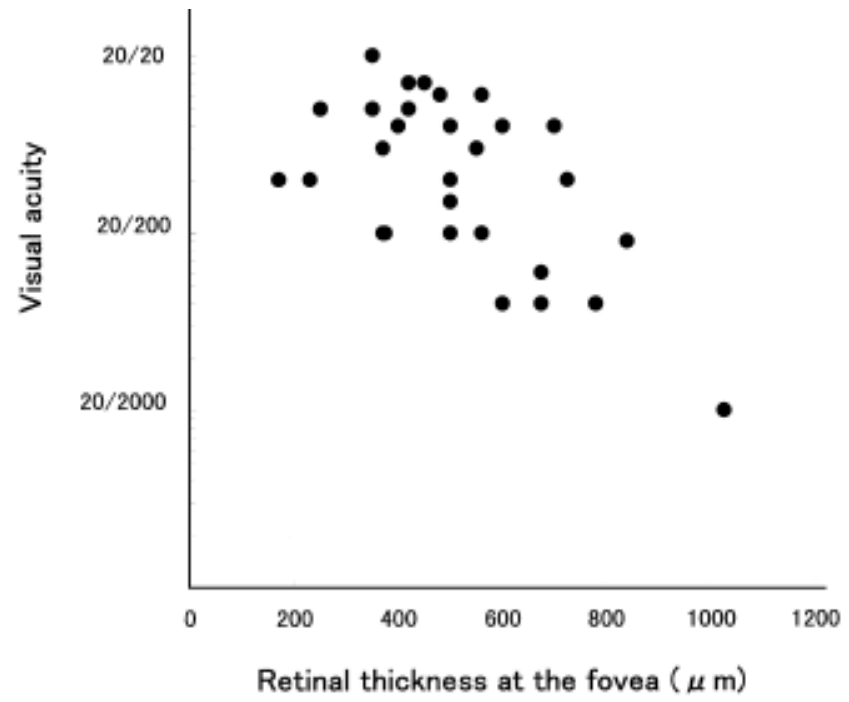

Fig. 13. Correlation between VA and retinal thickness at the fovea in patients with CME. Correlation coefficient $=-0.64, \mathrm{P}<0.01$. Figure from Otani, et al., 1999 .

When compared with FA, OCT findings also correlated well with the pattern of edema noticed in FA. Diffuse leakage is associated with nonspecific retinal swelling in the OCT image, whereas petaloid-pattern hyper fluorescence is associated with large cystic spaces in outer plexiform layer (OPL) and outer nuclear layer (ONL) and honeycomb hyper fluorescence is associated with cystic changes in the OPL, ONL, inner nuclear layer (INL) and inner plexiform layer (IPL) (Al-latayfeh et al., 2010). 


\subsubsection{Age related Macular Degeneration (AMD)}

OCT has proven extremely useful in both the diagnosis and treatment of AMD. It is even more so in the initial diagnosis of AMD lesion, as it is less hindered by blood or lipid as it is in FA (Singh \& Kaiser, 2007). A study compared the diagnostic accuracy of OCT with that of FA in predicting the angiographic pattern of choroidal neovascularization, and found the sensitivity and specificity to be $94 \%$ and $89 \%$ respectively when stereo fundus images were added (Sandhu \& Talks, 2005).

OCT has been used to update the drusen classification system; one group identified 3 drusen patterns whereas another group identified 17 different patterns (Khanifar et al., 2008; Pieroni et al., 2006). Drusen were classified based on shape (concave, convex saw-toothed), reflectivity (low to high), homogeneity (present or absent, with or without core), and foci of hyper-reflectivity (present or absent). Soft indistinct drusen were mostly found to be convex and homogenous with medium internal reflectivity and without overlying hyper-reflective foci (Khanifar et al., 2008). Another study showed that basal laminar drusen exhibited a moderately hyper-reflective and saw-toothed pattern on OCT images, and there was an associated sub-retinal fluid in the macula which could be monitored by using automated segmentation software in OCT (Leng et al., 2009). Fig. 14 shows FA, infrared and OCT images of both eyes of a patient with familial drusen. The OCT images show a saw-tooth appearance of the RPE-Bruch's membrane complex consistent with the presence of basal laminar drusen seen on the corresponding FA and infrared images.

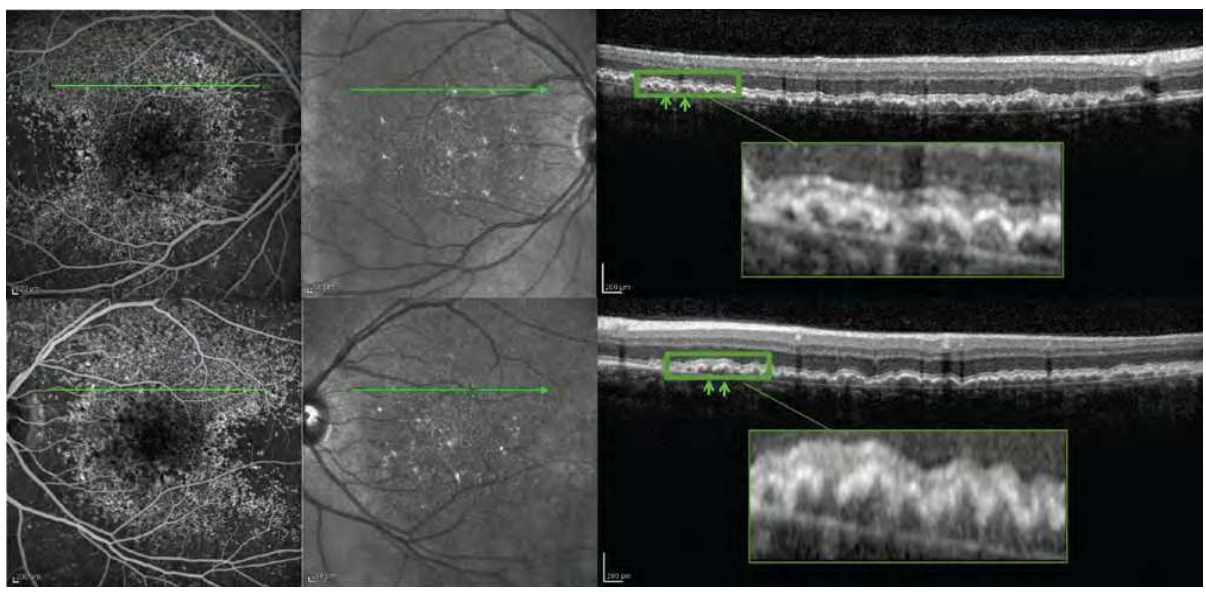

Fig. 14. Early-frame FA (left), infrared (center) and OCT (right) images of both eyes of a patient with familial drusen. Top row $=$ right eye, bottom row $=$ left eye. FA images show early hyperfluorescence characteristic of basal laminar drusen. Green line on FA and infrared images marks the area of OCT scans. In OCT images, the green arrows indicate a saw-tooth appearance of basal laminar drusen. Images from Sohrab, et al., 2011.

The effect of drusen on the overlying retina was investigated using OCT and it was found that the photoreceptor layer was thinned over $97 \%$ of drusen while inner retinal thickness was unaffected and over at least one druse in $47 \%$ of eyes, photoreceptor outer segments were absent. Furthermore, two types of hyper-reflective abnormalities were observed in the 
neurosensory retina over drusen: (1) distinct hyper-reflective speckled pattern over drusen (41\% of AMD eyes) and (2) prominent hyper-reflective haze in the photoreceptor nuclear layer over drusen (67\% of AMD eyes) (Schuman et al., 2009). Fig. 15 shows the two types of hyper-reflective abnormalities.

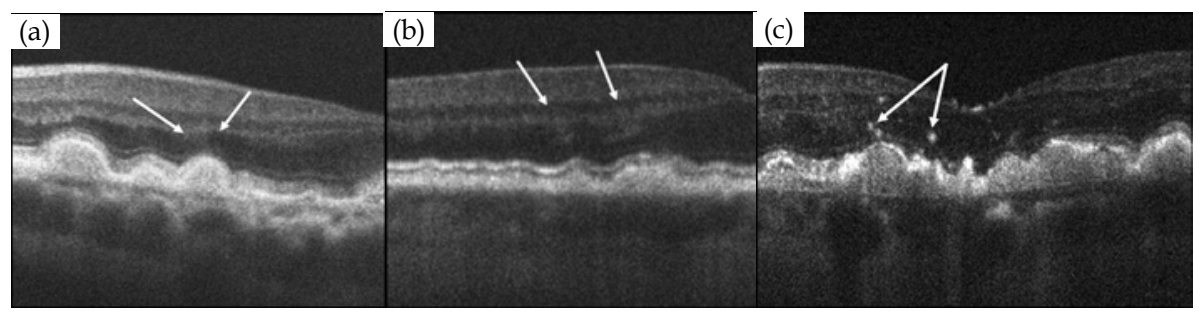

Fig. 15. $(a, b)$ The sites of prominent diffuse hyper-reflective haze were observed over drusen in 2 different eyes (shown with arrows). (c) Focal hyper-reflective speckling (arrows) was visible over drusen. Images from Schuman, et al., 2009.
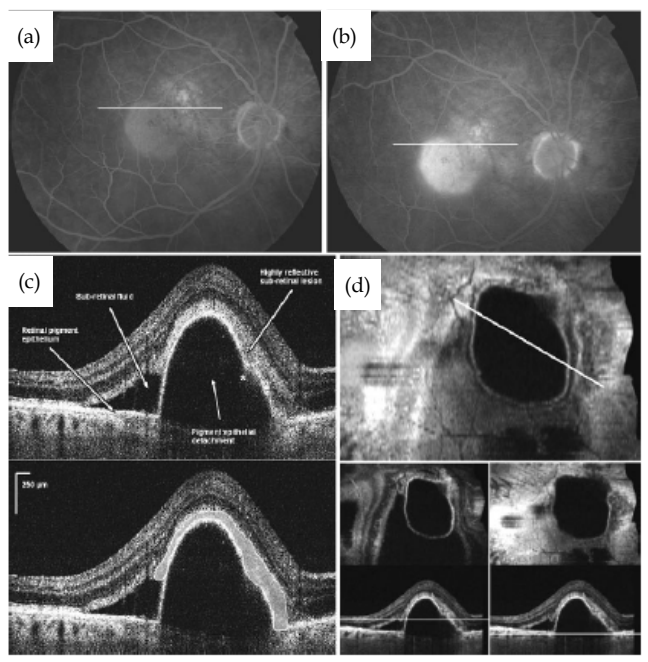

Fig. 16. FA and OCT images of the right eye in case 17. (a) Early-phase of FA. (b) Late-phase of FA. (c) OCT image (top) and the extent of the hyper-reflective lesion is outlined (bottom). (d) OCT C-scan was used to measure the greatest linear diameter (GLD) of the CNVM (top). OCT B-scans with white lines represent the cut depicted by the C-scan shown above (bottom). Images from Park, et al., 2010. Copyright [2010] ARVO.

The hyper-reflective foci overlying drusen are thought to represent progression of disease with RPE cell migration into the retina and possible photoreceptor degeneration or glial scar formation. The reduction in photoreceptor layer thickness suggests degeneration of photoreceptors which eventually leads to vision loss.

The choroidal neovascular membranes (CNVMs) have also been detected in all 21 eyes with exudative AMD by OCT and the anatomic growth pattern of the CNVM was determined based on the OCT images (Park et al., 2010). A highly reflective sub-retinal and/or sub-RPE 
lesion was visualized in the macula. Of twenty eyes, 7 eyes $(33 \%)$ had $>90 \%$ sub-RPE growth pattern (type 1), 10 eyes $(48 \%)$ had $>90 \%$ sub-retinal growth pattern (type 2 ) and 3 eyes $(14 \%)$ had a combined growth pattern. When the size of the CNVM was compared between FA and OCT images, a good correlation was found between these modalities (i.e., for classic CNVM, $r=0.99, \mathrm{P}<0.0001$ and for non-classic CNVM, $r=0.78, \mathrm{P}<0.001)$. Fig. 16 shows FA and OCT images from the right eye of a patient (case 17) with an occult CNVM associate with exudative AMD. The FA images showed hyper-fluorescence centered over the macula in the early-phase followed by an intense pooling of the dye into the pigment epithelial detachment (PED) with ill-defined leakage at the superonasal edge in the latephase. The OCT image showed a large PED with an adjacent area of sub-retinal fluid. A highly reflective sub-retinal lesion above the PED (consistent with type $2 \mathrm{CNVM}$ ) and a focal spot of discontinuity in the RPE (indicated with *) with a possible small extension of the hyper-reflectivity into the sub-RPE space were observed.

\subsubsection{Glaucoma}

OCT has shown the greatest potential for imaging glaucomatous structural changes such as the RNFL. A cross-sectional observational study involving 160 control subjects and 134 patients with primary open-angle glaucoma (POAG) was conducted to evaluate the accuracy of OCT in detecting differences in peripapillary RNFL thickness between normal and glaucomatous eyes as well as between different severity groups (Sihota et al., 2006). The POAG patients were divided into early $(n=61)$, moderate $(n=31)$, severe $(n=25)$ and blind $(n=17)$ groups. The OCT was reliable in detecting changes in the RNFL thickness between normal and all glaucoma subgroups. The RNFL thickness was significantly thinner in the POAG patients compared to the control subjects and also the RNFL thickness continued to reduce with an increase in the severity of POAG with $\mathrm{P}<0.001$.

The reproducibility of the RNFL thickness measurements was tested on 51 stable glaucoma patients using Stratus OCT (Budens et al., 2008). For the mean RNFL thickness, the intrasession and inter-session intraclass correlation coefficient (ICC) for the standard and fast scans were 0.98 and 0.96 respectively. The coefficient of variation (COV) ranged from 3.8 to $5.2 \%$ (Carpineto et al., 2003). Other study has reported lower ICC of 0.5 and higher COV of $10 \%$. However, the reproducibility is still sufficient to be useful clinically as a measure of glaucoma progression. Fig. 17 shows a picture of glaucomatous optic nerve head $(\mathrm{ONH})$ with a dense superior visual field defect. A circle concentric OCT scan around the ONH shows thinning of the RNFL in the inferiortemporal quadrant, which corresponds to both the visual field defect and loss of the neural rim of the ONH.
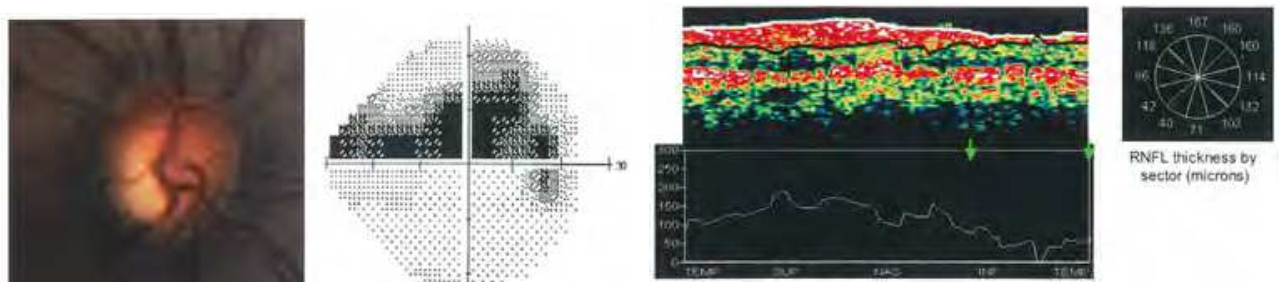

Fig. 17. An ONH picture, a visual field plot and a circle concentric OCT scan around the $\mathrm{ONH}$ from a glaucomatous eye. Green arrows indicate the area of thinning of the RNFL. Images from Jaffe, et al., 2004. 


\subsection{Adaptive optics - OCT}

With further improvement in lateral resolution of the system, it is possible to resolve even smaller details in the retina.

As previously mentioned, Kocaoglu et al. (2011) have shown the first measurements of RNFL axonal bundles (RNFB) in the living human eyes using their AO-OCT system. Four normal subjects and one subject with an arcuate RNFL defect were imaged and they were able to visualize individual RNFB in all subjects. Fig. 18 shows the individual RNFB in one of the normal subjects (S4). As the RNFB approach fovea, they become thinner and separate.
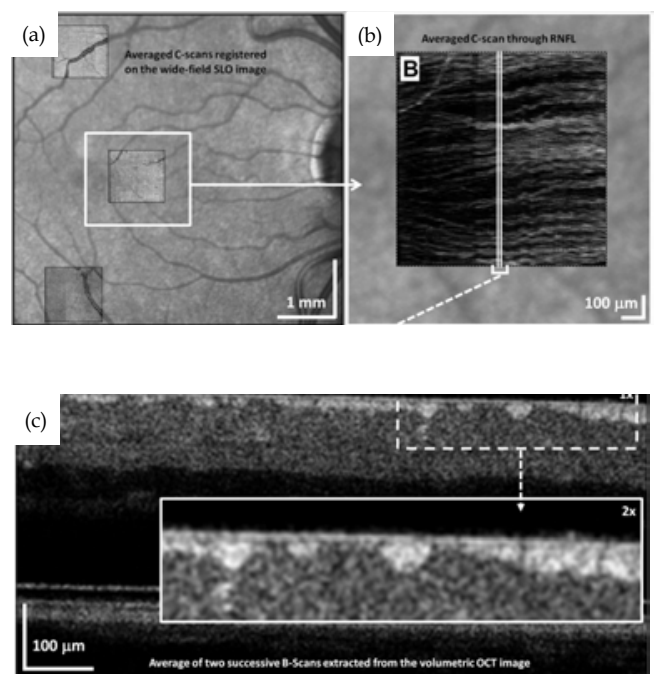

Fig. 18. (a) A wide field SLO, (b) An averaged C-scan and (c) An averaged B-scan images acquired from subject, $S 4$, at $3^{\circ}$ nasal retina (indicated with a white square). The white lines in (b) denote the area where the B-scans in (c) were obtained. The white solid rectangle in (c) represents $2 x$ magnification of the area outlined with white dotted rectangle. Images from Kocaoglu, et al., 2011.

In conjunction with the expected inner retinal changes such as, thinning of the RNFL in glaucoma and optic neuropathy, AO-OCT imaging has revealed outer retinal changes as well at the retinal locations with reduced visual function, specifically shortening of cone outer segments and blurring of the junction between the tip of the cone outer segments and RPE. These outer retinal changes only occurred when there was a permanent visual field loss. The same findings were observed in all types of optic neuropathy patients including glaucoma (Choi et al., 2008; Choi et al., 2011).

Fig. 19 shows AO-OCT images obtained from a nonarteritic anterior ischemic optic neuropathy (NAION) patient. The AO-OCT images were taken at 2 retinal locations, $2^{\circ}$ temporal $2^{\circ}$ superior retina and $4^{\circ}$ nasal $4^{\circ}$ superior retina. The $4^{\circ}$ nasal $4^{\circ}$ superior retina had better visual function than $2^{\circ}$ temporal $2^{\circ}$ superior retina, hence, the layer labeled 3 (the junction between the cone outer segment tip and RPE) was better defined and distinct at that location, and it was not visible at $2^{\circ}$ temporal $2^{\circ}$ superior retina. 


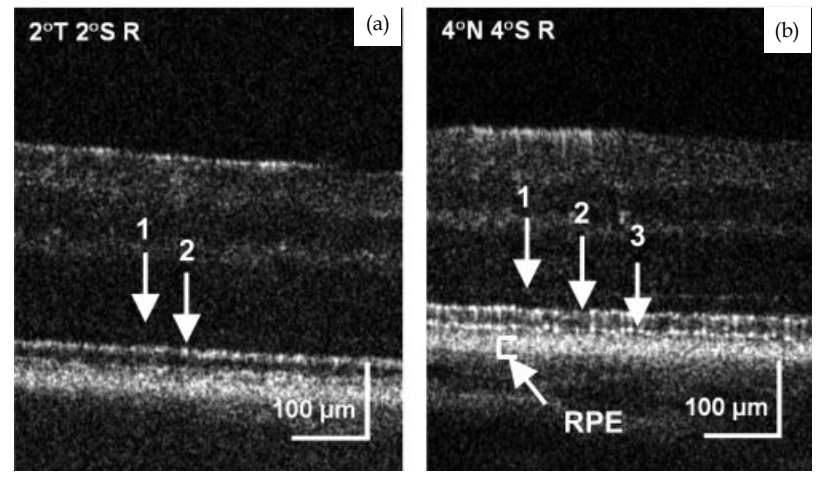

Fig. 19. AO-OCT images at two retinal locations in the right eye of the patient with NAION. (a) $2^{\circ}$ temporal $2^{\circ}$ superior retina and (b) $4^{\circ}$ nasal $4^{\circ}$ superior retina. 1: ELM, 2: IS/OS, 3 : OS/RPE. Images from Choi, et al., 2008. Copyright [2008] ARVO.

\section{Summary and future directions}

With technological advancements, OCT has undergone substantial changes towards high resolution cellular level OCT imaging. Light sources with $\geq 80 \mathrm{~nm}$ spectral bandwidth paved the way for sub-10 $\mu \mathrm{m}$ axial imaging. At the same time, the adoption of AO in OCT system's addressed ocular and systems aberrations, improving lateral resolutions. Various research groups have shown the potential of high resolution imaging with ultrawideband sources with $\mathrm{AO}$ in OCT systems. However for these systems to be widely implemented into the clinic, additional improvements are needed. Ultrawideband sources must provide sufficient power and stability, and are compact and inexpensive. Similarly, AO-OCT systems will also need to consider cost and size in addition to patient comfort and ease of use. Finally, by understanding and addressing the limitations of AO-OCT systems, can researchers and clinicians fully exploit the capabilities of $\mathrm{AO}$ to better image patients with various forms of retinal diseases.

The advent of OCT technology has allowed extensive amounts of new anatomic, physiologic, and pathologic data for the practice of ophthalmology. The ability to image retinal and ONH structures in detail in-vivo makes OCT an ideal modality for detection of early disease in screening and prevention for differential diagnosis of various diseases as well as for quantifying therapeutic effects and identifying recurrence during follow-up. The practicality of OCT in terms of obtaining motion artifact free images and presentation of quantified data in a user friendly way requires further improvement. Also, with an increase in the number of OCT users and commercial systems, there is a need to establish adequate normative data and common clinical standards to allow for consistency across devices and comparison between patients and diseases.

\section{Acknowledgment}

Financial support is acknowledged to the Department of Defense (DOD) Telemedicine and Advanced Technology Research Center (TATRC), W81XWH-10-1-0738. 


\section{References}

Adler, D.; Ko, T.; Konorev, A. et al. (2004). Broadband Light Source Based on Quantumwell Superluminescent Diodes for High-resolution Optical Coherence Tomography. Quantum Electronics, Vol. 34, No. 10, pp. 915-918, ISSN 10637818

Aguirre, A.; Nishizawa, N. \& Fujimoto, J. (2006) Continuum Generation in a Novel Photonic Crystal Fiber for Ultrahigh Resolution Optical Coherence Tomography at $800 \mathrm{~nm}$ and $1300 \mathrm{~nm}$. Opt Express, Vol. 14, No. 3, (Feb 2006), pp. 1145-1160, ISSN 10944087

Al-latayfeh, M.; Sun, J. \& Aiello, L. P. (2010). Ocular coherence tomography and diabetic eye disease. Semin Ophthal, Vol. 25, No. 5-6, (Sept - Nov, 12010), pp. 192-197, ISSN 17445205

Apolonski, A.; Povazay, B.; Unterhuber, A. et al. (2002) Spectral shaping of supercontinuum in a cobweb photonic-Crytal Fiber with Sub-20-fs-pulses. J Opt Soc Am A, B Vol. 19, No.9, (Sept 2002), pp.2165-2170, ISSN 1084-7529

Birks, T.; Wadsworth, J. \& Russel, P. (2000). Supercontinuum Generation in Tapered Fibers. Opt Letters, Vol. 25, No. 19, (Oct, 2000), pp. 1415-1417, ISSN 0146-9592

Budenz, D., Fredette, M., Feuer, W. et al. (2008). Reproducibility of peripapillary retinal nerve fiber thickness measurements with Stratus OCT in glaucomatous eyes. Ophthalmology, Vol. 115, No. 4, (Apr 2008), pp 661-666, ISSN 1549-4713

Carpineto, P.; Ciancaglini, M.; Zuppardi, E. et al. (2003). Reliability of nerve fiber layer thickness measurements using optic coherence tomography in normal and glaucomatous eyes. Ophthalmol, Vol. 110,No 1, (Jan 2003) pp 190-195, ISSN 15494713

Cense, B.; Koperda, E.; Brown, J. et al. (2009a). Volumetric Retinal Imaging with Ultra-high Resolution Spectral-Domain Optical Coherence Tomography and Adaptive Optics Using Two Broadband Light Sources. Opt Express, Vol. 17, No. 5, (Mar 2009), pp. 4095-4111, ISSN 1094-4087

Cense, B.; Gao, W.; Brown, J. et al., D.(2009b). Retinal Imaging with Polarization-Sensitive Optical Coherence Tomography and Adaptive Optics, Opt Express, Vol. 17. No. 24, (Nov 2009), pp. 21634-21651, ISSN 1094-4087

Choi, S.; Doble, N.; Lin, J. et al. (2005). Effect of Wavelength on in Vivo Images of the Human Cone Mosaic. J Opt Soc Am A, Vol. 22, No. 12, (Dec, 2005), pp. 2598-2605, ISSN 1084-7529

Choi, S.; Doble, N.; Hardy, J. et al. (2006) In vivo imaging of the photoreceptor mosaic in retinal dystrophies and correlations with visual function. Invest Ophthalmol Vis Sci, Vol. 47, No. 5, (May 2006), pp. 2080-2092, ISSN 1552-5783

Choi, S.; Zawadzki, R.; Keltner, J. et al. (2008). Changes in cellular structures revealed by ultra-high resolution retinal imaging in optic neuropathies. Invest Ophthalmol Vis Sci, Vol. 49, No. 5, (May 2008), pp. 2103-2119, ISSN 1552-5783

Choi, S.; Zawadzki, R.; Lim, M. et al. (2011). Evidence of outer retinal changes in glaucoma patients as revealed by ultrahigh-resolution in vivo retinal imaging. $\mathrm{Br} J$ Ophthalmol, Vol. 95, No. , (Jan 2011), pp. 131-141, ISSN 0007-1161 
Clivaz, X.; Marquis-Weible, F. \& Salathe, R. (1992). Optical Low Coherence Reflectometry with $1.9 \mu \mathrm{m}$ Spatial Resolution. Elect Letters, Vol. 28, No. 16, (Jul 1992), pp.15531555, ISSN 0013-5194

Coen, S. \& Haelterman, M. (2001). Continuous-wave Ultrahigh-repetition-rate Pulse-train Generation Through Modulational Instability in a Passive Fiber Cavity. Opt Letters, Vol. 26, No. 1, (Jan 2001), pp. 39-41, ISSN 0146-9592

de Boer, J.; Cense, B.; Park, B. et al. (2003). Improved Signal-to-Noise Ratio in Spectral Domain Compared with Time-Domain Optical Coherence Tomography. Opt Letters, Vol. 28, No. 21 , (Nov 2003), pp. 2067-2069, ISSN 0146-9592

Dreher, A.; Billie, J. \& Weinreb, R. (1989). Active Optical Depth Resolution Improvement of the Laser Tomographic Scanner. Appl Opt, Vol. 28, No. 4, (Apr 1989), pp. 804-808, ISSN 0003-6935

Drexler, W.; Mogner, U.; Kartner, F et al. (1999). In vivo Ultrahigh-resolution Optical Coherence Tomography. Opt Letters, Vol. 24, No. 17, (Sept 1999), pp. 1221-1223, ISSN 0146-9592

Drexler, W.; Morgner, U.; Ghanta, R. et al. (2001). Ultrahigh-resolution Ophthalmic Optical Coherence Tomography. Nat Med, Vol. 7, No. 4, (Apr 2001), pp. 502-507, ISSN 1078-8956

Drexler, W.; Sattman, H.; Hermann B. et al. (2003). Enhanced Visualization of Macular Pathology With the Use of Ultrahigh-Resolution Optical Coherence Tomography. Arch of Ophthalmol, Vol. 121, No. 5, (May 2003), pp. 695-706, ISSN 0003-9950

Drexler, W. (2004). Ultrahigh-resolution Optical Coherence Tomography. J Biomed Opt, Vol .9, No. 1, (Jan/Feb 2004), pp. 47-74, ISSN 1083-3668

Doble, N. \& Miller, D. (2006). Wavefront Correctors for Vision Science, In: Adaptive Optics for Vision Science, J. Porter, H. Queener, J. Lin, K. Thorn, A. Awwal (Ed) , pp. 82-117, ISBN 978-0-471-67941-7, Hoboken, NJ, USA.

Fercher, A.; Mengedoht, K. \& Werner, W. (1988) Eye-length Measurement by Interferometry with Partially Coherent Light. Opt Letters, Vol. 13, No. 3, (Mar 1988), pp. 186-188, ISSN 0146-9592

Fernandez, E.; Iglesias, I. \& Artal, P. 2001. Closed-loop Adaptive Optics in the Human Eye. (2001). Opt Express, Vol. 26, No. 10, (May, 2001), pp. 746-748, ISSN 0146-9592

Ferguson, R.; Hammer, D.; Paunescu, L. et al. Tracking Optical Coherence Tomography. Opt Letters, Vol. 29, No. 18, (Sept 2004), pp. 2139-2141, ISSN 0146-9592

Fujimoto, J; De Silvestri, S.; Ippen, E. et al. (1986). Femtosecond Optical Ranging in Biological Systems. Opt Letters, Vol. 11, No. 3, (Mar 1986), pp. 150-152, ISSN 01469592

Hartl, I.; Li, X.; Chudoba, C. et al. (2001). Ultrahigh-resolution Optical Coherence Tomography Using Continuum Generation in a Air-Silica Microstructure Optical Fiber. Opt Letters, Vol. 26, No. 9, (May 2001), pp. 608-610, ISSN 0146-9592

Hee, M.; Puliafifito C.; Duker, J. et al. (1998). Topography of diabetic macualr edema with optical coherence tomography. Ophthalmology, Vol. 105, No. 2, (Feb 1998), pp. 360370, ISSN 1549-4713 
Hofer, H.; Chen, L.; Yoon, G. et al. (2001). Improvement in Retinal Image Quality with Dynamic Correction of the Eye's Aberrations. Opt Express, Vol. 8, No. 11, (May 2001), pp. 631-643, ISSN 0146-9592

Hong, Y.;Makita, S.; Yamanari, M. et al. (2007). Three-dimensional Visualization of Choroidal Vessels by Using SStandard and Ultra-high Resolution Optical Coherence Tomography. Opt Express, Vol. 15, No. 12, (Jun 2007), pp. 7538-7550, ISSN 0146-9592

Huang, D.; Swanson, E.; Lin, C. et al. (1991). Optical Coherence Tomography. Science, Vol. 254, No.5035, (Nov 1991), pp.1178-1181, ISSN 0036-8075

Humbert, G.; Wadsworth, W.; Leon-Saval, G. et al. (2006). Supercontinuum Gneration System for Optical Coherence Tomography based on Tapered Photonic Crytal Fibre. Opt Express, Vol. 14, No. 4, (Feb 2006), pp. 1596-1603.

Jaffe, G. J. \& Caprioli, J. (2004). Optical coherence tomography to detect and manage retinal disease and glaucoma. Am J Ophthalmol, Vol. 137, No. 1, (Jan 20044), pp. 156-169, ISSN 0002-9394

Jonall, R.; Rha, J.; Zhang, Y et al. (2007). In Vivo Functional Imaging of Human Cone Photoreceptors. Opt Express, Vol. 15, No. 24, (Nov 2007), pp 16141-16160, ISSN 0146-9592

Jungtae, R.; Jonnal, R.; Thorn, K., et al. (2006). Adaptive Optics Flood-Illumination Camera for High Speed Retinal Imaging. Opt Express, Vol. 14. No. 10, (May 2006), pp. 45524569, ISSN 0146-9592

Khanifar, A.; Koreishi, A.; Izatt, J. et al. (2008). Drusen ultrastructure imaging with spectral domain optical coherence tomography in age-related macular degeneration. Ophthalmology, Vol. 115, No. 11, (Nov 2008), pp.1883-1890, ISSN $1549-4713$

Ko, T.; Adler, D.; Fujimoto, J. et al.. (2004). Ultrahigh Resolution Optical Coherence Tomography Imaging with a Broadband Superluminscent Diode Light Source. Opt Express, Vol 12, No. 10, (May 2004), pp. 2112-2119, ISSN 1094-4087

Kocaoglu, O.; Cense, B.; Jonnal, R. et al. (2011). Imaging Retinal Nerve Fiber Bundles Using Optical Coherence Tomography with Adaptive Optics. Vision Res, Vol. 51, No. 16, (Aug 2011), pp. 1835-1844, ISSN 42-6989

Leng, T.; Rosenfeld, P.; Puliafito, G. et al. (2009). Spectral domain optical coherence tomography characteriatics of cuticular drusen. Retina, Vol. 29, No. 7, (Jul-Aug 2009),pp. 988-993, ISSN 1539-2864

Liang, J.; Williams, D.; \& Miller, D. (1997). Supernormal Vision and High-Resolution Retinal Imaging Through Adaptive Optics. J Opt Soc Am A, Vol. 14 No. 11, (May, 2007), pp. 2884-2892, ISSN 1084-7529

Lietgeb, R.; Hitzenberger, C. \& Fercher, A. (2003). Performance of Fourier Domain vs. Time Domain Optical Coherence Tomography. Opt Express, Vol. 11, No. 8, (Apr 2003), pp.889-894, ISSN 0146-9592

Mujat, M.; Ferguson, D.; Patel, A. et al. (2010). High Resolution Multimodal Clinical Ophthalmic Imaging System. Opt Express, Vol. 18, No. 11, (May 2010), pp. 1160711621, ISSN 0146-9592 
Ontani, T.; Kishi, S. \& Maruyama, Y. (1999) Patterns of diabetic macular edema with optical coherence tomography. Am J Ophthalmol, Vol. 127, No. 6,(Jun 1999) pp. 688-693, ISSN 0002-9394

Park, S.; Truong, S., Zawadzki, R. et al. (2010). High-resolution Fourier-domain Optical Coherence Tomography of Choroidal Neovascular Membranes Associated with Age-related Macular Degeneration. Invest Ophthalmol Vis Sci., Vol. 51, No. 8, (Aug 2010), pp. 4200-4206, ISSN 1552-5783

Pieroni, C.; Witkin, A.; Ko, $\mathrm{T}$ et al. (2006). Ultrahigh resolution optical coherence tomographyin non-exudative age-related macular degeneration. Br J Ophthalmol, Vol. 90, No. 2, (Feb 2006), pp. 191-197, ISSN 0007-1161

Potsaid, B.; Gorczynska, I.; Srinivasan, V. et al. (2008). Ultrahigh Speed Spectra/Fourier Domain OCT Opthalmic Imaging at 70,000 to 312,500 Axial Scans per Second. Ophthalmol Express, Vol. 16, No. 19, (Sept 2008), pp. 15149-15169, ISSN 0146-9592

Povazay, B.; Bizheva, K.; Hermann, A. et al. (2003). Enhanced Visualization of Choroidal Vessels using Ultrahigh Resolution Ophthalmic OCT at $1050 \mathrm{~nm}$. Opt Express, Vol. 11, No. 17, (Aug 2003), pp. 1980-1986, ISSN 0146-9592

Puvanathasan, P.; Forbes, P.; Ren, Z. et al. (2008). High-speed, high resolution Fourierdomain optical coherence tomography system for retinal imaging in the $1060 \mathrm{~nm}$ wavelength region. Opt Letters, Vol. 21, No. 21, (Nov 2008) pp. 2479-2481, ISSN 0146-9592

Roorda, A. \& Williams D. (2002a). Optical Fiber Properties of Individual Human Cones. J Vis, Vol. 2, No. 5, (Apr, 2002), pp. 404-412, ISSN 1534-7362

Roorda, A.; Romero-Borja, F.; Donnely, W. et al. Adaptive Optics Laser Scanning Opthalmoscopy. (2002b). Opt Express, Vol. 10, No. (May 2002), pp. 405-412, ISSN 0146-9592

Sandhu, S. \& Talks S. (2005). Correlation of optical coherence tomography, with or without additional colour fundus photography, with stereo fundus fluorescein angiography in diagnosing choroidal neovascular membranes. Br J Ophthalmol, Vol. 89, No. 8, (Aug 2005), pp. 967-970, ISSN 0007-1161

Schocket, L.; Wikins A.; Fujimoto, J. et al. (2006). Ultrahigh-Resolution Optical Coherence Tomography in Patients with Decreased Visual Acuity after Retinal Detachment Repair. Ophthalmol, Vol. 113, No. 4, (Apr 2006), pp. 666-692, ISSN 0161-6420

Schuman, S.; Koreishi, A.; Farsiu, S. et al. (2009). Photoreceptor layer thinning over drusen in eyes with age-related macular degeneration imaged in vivo with sepctral domain optical coherence tomography. Ophthalmol, Vol. 116, No. 3, (Mar 2009), pp. 488-496, ISSN 1549-4713

Sihota, R.; Sony, P.; Gupta, V. et al. (2006). Diagnostic Capability of Optical Coherence Tomography in Evaluating the Degree of Glaucomatous Retinal Nerve Fiber Damage. Invest Ophthalmol Vis Sci, Vol. 47, No. 5, (), pp 2006-2010, , ISSN 15525783

Singh, R. \& Kaiser, P. K. (2007). Advances in AMD imaging. Int Ophthalmol Clin, Vol. 47, No. 1 (Winter 2007), pp. 65-74, ISSN 0020-8167 
Spoler, F.; Kray, S.; Grychtol, P. et al. (2007). Simultaneous Dual-band Ultra-high Resolution Optical Coherence Tomography. Opt Express, Vol. 15, No. 17, (Aug 2007), pp. 10832-10841, ISSN 1094-4087

Srinivasan, V.; Monson, B.; Wojtkowski, M. et al. (2006). High-Definition and 3-dimensional Imaging of Macular Pathologies with High-speed Ultrahigh-Resolution Optical Coherence Tomography, Ophthalmology, Vol. 113, No. 11, (Nov 2006), pp. 20542065, ISSN 1549-4713

Srinivasan, V.; Monson, B.; Wojtkowski, M. et al. (2008). Characterization of Outer Retinal Morphology with High-Speed, Ultrahigh-Resolution Optical Coherence Tomography. Invest Ophthalmol Vis Sci, Vol. 49, No. 4, (Apr 2008), pp. 1571-1579, ISSN 0146-0404

Swanson, E.; Izatt, J.; Hee, M. et al. (1993). In Vivo Retinal Imaging by Optical Coherence Tomography. Opt Letters, Vol. 18, No. 21,(Nov 1993), pp. 1864-1866, ISSN 01469592

Tao, Y.; Farsiu, S. \& Izatt, J. (2010). Interlaced Spectrally Encoded Confocal Scanning Laser Ophthalmoscopy and Spectral Domain Optical Coherence Tomography. Biomed Opt Express, Vol. 1, No. 2, (Sept 2010), pp. 431-440, ISSN 0146-9592

Unterhuber, A.; Povazay, B; Hermann, B. et al. (2003). Compact, Low-cost Ti:Al2O3 Laser for In Vivo Ultrahigh-resolution Optical Coherence Tomography. Opt Letters, Vol. 28, No. 11 (Jun 2003), pp. 905-907, ISSN 0146-9592

Witkin, A.; Ho, T.; Fujimoto, J, et al. (2006). Ultra-high Resolution Optical Coherence Tomography Assessment of Photoreceptros in Retinintis Pigmentosa and Related Diseases. Am J Ophthalmol, Vol. 142, No. 6, (Dec 2006), pp.945-952, ISSN 00029394

Wolfing, J.; Chung, M.; Carroll, J. et al. (2006). High Resolution Imaging of Cone-Rod Dystrophy with Adaptive Optics. Ophthalmology, Vol. 113, No. 6, (Jun 2006), pp. 1014-101, ISSN 1549-4713

Xue, B.; Choi, S.; Doble, N. et al. (2007). Photoreceptor counting and montaging of en-face retinal iamges from an adaptive optics fundus camera. J Opt Soc Am A, Vol. 24, Issue 5, (Apr 2007) , pp. 1364-1372, ISSN 1084-7529

Zawadazki, R.; Jones, S.; Olivier, S. et al. (2005). Adaptive-Optics Optical Coherence Tomography for High-Resolution and High-Speed 3D Retinal In Vivo Imaging. Opt Express, Vol. 13, No. 21, (Oct 2005), pp. 8532-8546, ISSN 0146-9592

Zawadzki, R.; Choi, S.; Olivier, S. et al. (2007). J Opt Soc Am A, Vol. 24, Issue 5, (May 2007) , pp. 1373-1383, ISSN 1084-7529

Zawadzki, R.; Cense, B.; Zhang, Y. et al. (2008). Ultrahigh-resolution Optical Coherence Tomography with Monochromatic and Chromatic Aberration Correction. Opt Express, Vol. 16, No. 11, (May 2008), pp. 8126-8143, ISSN 1094-4087

Zawadazki, R., Jones, S., Chen, D. et al. (2009). Combined Adaptive Optics - Optical Coherence Tomography and Adaptive Optics - Scanning Laser Ophthalmoscopy System for Retinal Imaging, Proceed of SPIE, ISBN 9780819474094, San Jose, CA, USA, Jan 24, 2009 
Zawadzki, R.; Jone, S.; Pilli, S.; Balderas-Mata, S.; Kim, D.; Olivier, S. \& Werner, J. (2011) Integrated Adaptive Optics Optical Coherence Tomography and Adaptive Optics Scanning Laser Ophthalmoscope System for Simultaneous Cellular Resolution In Vivo Retinal Imaging. Biomed Opt Express, Vol. 2, No. 6, (May 2011), pp. 1674-1686, ISSN 0146-9592 


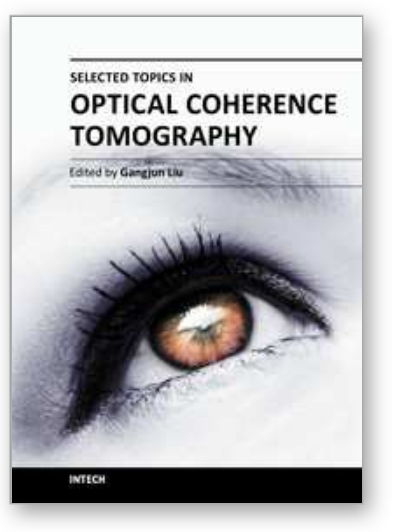

\author{
Selected Topics in Optical Coherence Tomography \\ Edited by Dr. Gangjun Liu
}

ISBN 978-953-51-0034-8

Hard cover, 280 pages

Publisher InTech

Published online 08, February, 2012

Published in print edition February, 2012

This book includes different exciting topics in the OCT fields, written by experts from all over the world. Technological developments, as well as clinical and industrial applications are covered. Some interesting topics like the ultrahigh resolution OCT, the functional extension of OCT and the full field OCT are reviewed, and the applications of OCT in ophthalmology, cardiology and dentistry are also addressed. I believe that a broad range of readers, such as students, researchers and physicians will benefit from this book.

\title{
How to reference
}

In order to correctly reference this scholarly work, feel free to copy and paste the following:

Cherry Greiner and Stacey S. Choi (2012). Cellular Level Imaging of the Retina Using Optical Coherence Tomography, Selected Topics in Optical Coherence Tomography, Dr. Gangjun Liu (Ed.), ISBN: 978-953-510034-8, InTech, Available from: http://www.intechopen.com/books/selected-topics-in-optical-coherencetomography/cellular-level-imaging-of-the-retina-using-optical-coherence-tomography

\section{INTECH}

open science | open minds

\author{
InTech Europe \\ University Campus STeP Ri \\ Slavka Krautzeka 83/A \\ 51000 Rijeka, Croatia \\ Phone: +385 (51) 770447 \\ Fax: +385 (51) 686166 \\ www.intechopen.com
}

\author{
InTech China \\ Unit 405, Office Block, Hotel Equatorial Shanghai \\ No.65, Yan An Road (West), Shanghai, 200040, China \\ 中国上海市延安西路65号上海国际贵都大饭店办公楼 405 单元 \\ Phone: +86-21-62489820 \\ Fax: $+86-21-62489821$
}


(C) 2012 The Author(s). Licensee IntechOpen. This is an open access article distributed under the terms of the Creative Commons Attribution 3.0 License, which permits unrestricted use, distribution, and reproduction in any medium, provided the original work is properly cited. 